\title{
ARTICLE
}

\section{Imaging and controlling plasmonic interference fields at buried interfaces}

\author{
Tom T.A. Lummen', Raymond J. Lamb², Gabriele Berruto1, Thomas LaGrange ${ }^{3}$, Luca Dal Negro4, \\ F. Javier García de Abajo ${ }^{5,6}$, Damien McGrouther ${ }^{2}$, B. Barwick ${ }^{7}$ \& F. Carbone ${ }^{1}$
}

Capturing and controlling plasmons at buried interfaces with nanometre and femtosecond resolution has yet to be achieved and is critical for next generation plasmonic devices. Here we use light to excite plasmonic interference patterns at a buried metal-dielectric interface in a nanostructured thin film. Plasmons are launched from a photoexcited array of nanocavities and their propagation is followed via photon-induced near-field electron microscopy (PINEM). The resulting movie directly captures the plasmon dynamics, allowing quantification of their group velocity at $\sim 0.3$ times the speed of light, consistent with our theoretical predictions. Furthermore, we show that the light polarization and nanocavity design can be tailored to shape transient plasmonic gratings at the nanoscale. This work, demonstrating dynamical imaging with PINEM, paves the way for the femtosecond and nanometre visualization and control of plasmonic fields in advanced heterostructures based on novel two-dimensional materials such as graphene, $\mathrm{MoS}_{2}$, and ultrathin metal films.

\footnotetext{
${ }^{1}$ Laboratory for Ultrafast Microscopy and Electron Scattering, ICMP, École Polytechnique Fédérale de Lausanne, Station 6, CH-1015 Lausanne, Switzerland. ${ }^{2}$ SUPA, School of Physics and Astronomy, University of Glasgow, Glasgow G12 8QQ, UK. ${ }^{3}$ Interdisciplinary Center for Electron Microscopy (CIME), École Polytechnique Fédérale de Lausanne, CH-1015 Lausanne, Switzerland. ${ }^{4}$ Department of Electrical and Computer Engineering and Photonics Center, Boston University, 8 Saint Mary's Street, Boston, Massachusetts 02215, USA. ${ }^{5}$ ICFO - Institut de Ciencies Fotoniques, The Barcelona Institute of Science and Technology, Castelldefels, 08860 Barcelona, Spain. ${ }^{6}$ ICREA - Institució Catalana de Recerca i Estudis Avancats, Passeig Lluís Companys, 23, Barcelona 08010, Spain. ${ }^{7}$ Department of Physics, Trinity College, 300 Summit Street, Hartford, Connecticut 06106, USA. Correspondence and requests for materials should be addressed to T.T.A.L. (email: tom.lummen@epfl.ch) or to F.C. (email: fabrizio.carbone@epfl.ch).
} 
S urface plasmon polaritons (SPPs) ${ }^{1}$ are considered ideal for circuitry miniaturization to nanoscale dimensions ${ }^{2}$, due to their inherent surface-bound and spatially confined characteristics. This makes them appealing for various applications, such as nanoscopic signal processing ${ }^{3}$ and high-resolution sensing ${ }^{4}$. However, considering the femtosecond (fs) temporal and nanometre $(\mathrm{nm})$ spatial scales involved, direct real space characterization of evanescent waves presents a significant experimental challenge, especially in materials and devices involving buried interfaces and complex heterostructures.

Several optics-based approaches have been developed to meet the challenge of tracking surface-bound SPPs with appropriate resolution, notably dual-colour coherent anti-Stokes Raman scattering $(\mathrm{CARS})^{5}$ and scanning-tip-based techniques such as near-field scanning optical microscopy (NSOM $)^{6-8}$. In recent years, the spatial and temporal resolution limits of these optical techniques have been pushed to the tens of $\mathrm{nm}$ and hundreds of $\mathrm{fs}^{9,10}$. In an alternative approach, the use of electron photoemission for detection, as in time-resolved nonlinear photoemission electron microscopy (TR-PEEM) ${ }^{11-13}$, has allowed for comparable spatial resolution in imaging SPPs, with a time resolution extending down to the few-fs single optical cycle regime. Recent advances in TR-PEEM methods have facilitated the successful spatiotemporal mapping of localized and propagating surface plasmons in a variety of systems ${ }^{14-18}$, even allowing for experimental access to plasmon phases ${ }^{19,20}$. Furthermore, both NSOM and TR-PEEM have demonstrated sensitivity towards probing the near-field corresponding to SPPs confined to subsurface interfaces with a spatial resolution of up to $40 \mathrm{~nm}$ (refs 19,21-23). However, as TR-PEEM relies on sample-emitted photoelectrons, and scanning-tip-based techniques require physical probe proximity to the evanescent field, both are inherently limited to the study of electromagnetic near-fields that are external to materials. In addition, the connection between the probed field and the local density of photonic states is not always direct $^{19,24}$, although PEEM measurements performed in a nonconventional normal incidence geometry largely overcome this issue $e^{25}$. Due to these restrictions, these techniques are not suited for studies of advanced multilayer systems and heterostructured devices that rely on truly sub-surface plasmonic waves bound to buried interfaces.

An alternative method to those mentioned above employs electron energy loss spectroscopy (EELS) in a transmission electron microscope ${ }^{26}$, either in a spectrum imaging or energyfiltered transmission electron microscopy configuration, enabling $\sim 1 \mathrm{~nm}$ spatial resolution in the imaging of SPPs in thin films and nanostructures ${ }^{27-29}$. In these experiments, plasmonic guided modes are excited by the transient electric field generated by the fast $(80-300 \mathrm{keV})$ electrons traversing the specimen $^{30,31}$. In its most widespread spectrum imaging implementation, EELS is combined with an electron beam raster scanning approach, offering temporal imaging resolution down to the millisecond (camera-rate) range. Recently, a new technique termed photon-induced near-field electron microscopy $(\mathrm{PINEM})^{32}$ extended the accessible domain of EELS-based experiments to the $\mathrm{nm} / \mathrm{fs}$ regime by optically exciting SPPs with intense fs laser pulses, and subsequently probing the resulting plasmonic near-fields through their interaction with ultrafast electron bunches ${ }^{33-35}$. By virtue of the employed transmission geometry, the SPP near-field is probed along the entire trajectory of these relativistic electrons, which couple to the field component along the electron propagation direction ${ }^{35,36}$. By creating an image using only the transmitted electrons that have gained energy through the inelastic scattering by plasmons, PINEM performed in the energy-filtered transmission electron microscopy configuration enables the direct field-of-view imaging of photoexcited plasmonic fields ${ }^{37,38}$. Compared to CARS, NSOM and TR-PEEM, the PINEM approach is able to directly access the sub-surface plasmonic near-field of the SPPs bound to buried interfaces, even when such fields are entirely internal to the heterostructure. This is possible while maintaining a proven sub-10 nm spatial resolution (Supplementary Fig. 1), with a $1 \mathrm{~nm}$ resolution readily available when further optimized ${ }^{37}$. The noninvasive probe does not strongly affect the plasmons being measured and couples exclusively to the evanescent component of the electromagnetic field at the sample. It thus avoids incident or reflected beam signal contributions and enables a direct image interpretation. PINEM further allows for the tailored study of specific plasmons in any energy range, due to its complete decoupling of the optical excitation and the electron probing mechanisms.

These advantages, along with the convenient ability to obtain complementary nanoscale morphological, structural and chemical information through static or time-resolved TEM imaging, electron diffraction and EELS in the same setup, make PINEM uniquely suited for probing plasmonics in more complex geometries and advanced multilayer systems. In recent years, PINEM has been used to image optical near-fields at the surfaces of a variety of materials, nanostructures and biological specimens at energies ranging from near ultraviolet to infrared $32,37-41$, although these efforts did not track the dynamical behaviour of the photoinduced plasmons or target the material properties of the specimens. So far, the use of PINEM to experimentally access SPP dynamics has remained an open challenge due to the typical SPP lifetime (on the order of $100 \mathrm{fs}$ ) being beyond instrumental temporal resolution (to date at best $\sim 200 \mathrm{fs}$ using $\sim 60 \mathrm{fs}$ single optical pulses (Supplementary Fig. 2), extendable down to the optical pulse durations through photon gating ${ }^{42}$ ), with the latter being primarily limited by the electron bunch duration $^{38}$. Given the recent progress in the generation of attosecond electron bunches, there is a lot of potential to improve the time resolution of the PINEM approach even further ${ }^{40}$.

Here we employ localized, embedded SPP sources to launch SPPs in a two-dimensional (2D) plasmonic waveguide, provided by a buried $\mathrm{Ag} / \mathrm{Si}_{3} \mathrm{~N}_{4}$ interface, allowing the photoinduced plasmons to propagate and interfere over several microns. In this geometry, due to the finite time delay needed for plasmon propagation, the scattering of probe electrons by the propagating SPP wave occurs at later times as the distance to the source increases. By visualizing and characterizing this temporal dependence using time-resolved PINEM imaging, we could follow the dynamical evolution of the collective plasmonic field propagating at a buried interface with combined ultrafast and nanoscale resolution.

\section{Results}

Capturing plasmon dynamics. A conceptual scheme of our experiment is sketched in Fig. 1a. A linearly polarized optical pump pulse is incident on a nanocavity in a $\mathrm{Si}_{3} \mathrm{~N}_{4}$-supported $\mathrm{Ag}$ thin film, launching SPPs of energy $E_{\mathrm{SPP}}=\hbar \omega$ that are confined to the $\mathrm{Ag} / \mathrm{Si}_{3} \mathrm{~N}_{4}$ interface. At a variable time delay $\tau$, the propagating plasmonic wave is then probed by a spatially dispersed pulse of fast electrons, which inelastically scatter from the optically excited SPPs. This scattering interaction results in the electrons gaining and losing integer numbers of SPP energy quanta $33,34,36,40$, yielding equidistantly spaced peaks in the energy distribution of the transmitted electrons. By subsequently constructing an energy-filtered image using only those electrons that have gained energy, one directly obtains a spatially resolved snapshot of the $E_{Z}$ component of the buried plasmonic near-field ${ }^{32,38}$. Due to the large field of view of the electron 


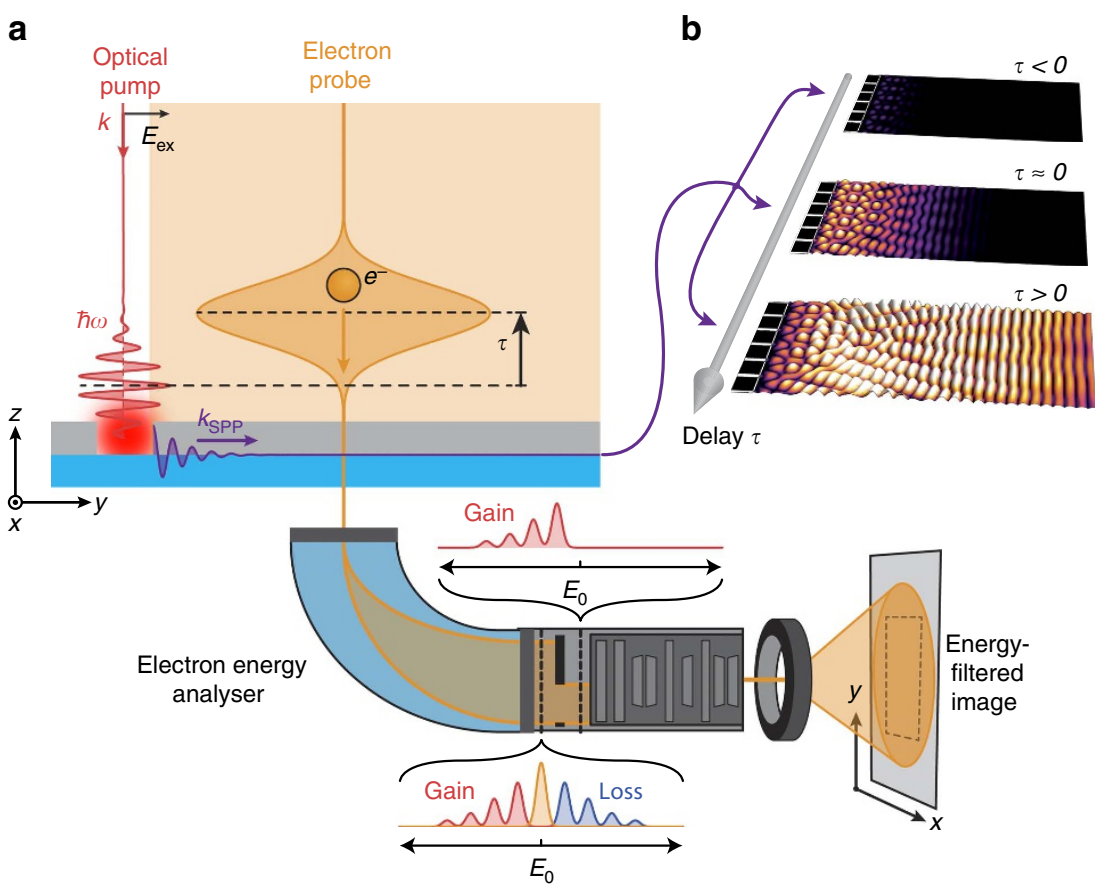

Figure 1 | Time-resolved PINEM methodology. (a) Simplified scheme of the time-resolved photon-induced near-field electron microscopy (PINEM) experiments in this work. A photon pump pulse incident on a nanopatterned $\mathrm{Ag}$-on- $\mathrm{Si}_{3} \mathrm{~N}_{4}$ bilayer structure generates a surface plasmon polariton (SPP) wave propagating along the buried $\mathrm{Ag} / \mathrm{Si}_{3} \mathrm{~N}_{4}$ interface. The near-field of the propagating SPP is subsequently probed through its interaction with a field-of-view electron pulse at a time delay $\tau$. Energy-filtered imaging of the resulting electron distribution of transmitted electrons then provides spatially resolved temporal snapshots of the near-field corresponding to the propagating plasmonic wave. (b) Variation of the relative time delay between the optical excitation pulse and the probing electron pulse generates a time-resolved movie of the ultrafast evolution of the buried plasmonic near-field

beam employed here, snapshots recorded at different relative time delays $\tau$ capture the spatiotemporal evolution of the photoinduced plasmonic wave (Fig. 1b).

The sample studied in this work consisted of a $30 \mathrm{~nm} \mathrm{Ag}$ thin film deposited on a $50 \mathrm{~nm} \mathrm{Si} \mathrm{N}_{4}$ membrane. A variety of different rectangular nanocavity (NC) arrays were patterned into the Ag film using focused ion beam milling (Methods and Supplementary Fig. 3). To experimentally measure the propagation speed of SPPs at the buried interface, we monitor a large, featureless area adjacent to a linear array of NCs (Fig. 2a). The optical pump beam (diameter $\simeq 100 \mu \mathrm{m}, \lambda_{0}=786$ $\mathrm{nm}$, full-width-half-maximum (FWHM) duration $\sim 105 \mathrm{fs}$ ) and the photoelectron probe beam (diameter $\simeq 40 \mu \mathrm{m}, E_{0}=200 \mathrm{keV}$, FWHM duration $\sim 650 \mathrm{fs}$ ) are spatially overlapped at nearnormal incidence on the $\mathrm{Ag}$ side of the nanopatterned bilayer sample. The optical pump pulses generate SPPs at the edges of the $\mathrm{NCs}$, with each edge coupling to the incident electric field component along the in-plane edge normal ${ }^{43}$. Since the NCs fully perforate the metal film, photoexcited SPPs are transmitted through the NCs to the other side of the Ag layer and subsequently launched across the $\mathrm{Ag} / \mathrm{Si}_{3} \mathrm{~N}_{4}$ interface $^{44}$. In the first approximation, each NC edge effectively acts as a source of SPPs that travel radially outward in a point-dipole-like pattern at the buried interface ${ }^{45}$. Consequently, according to the Huygens-Fresnel principle, the interferometric plasmonic wave propagating away from the NC array (along the $y$ axis in Fig. 2a) corresponds to the coherent superposition of the SPPs launched from the different NCs.

Figure $2 \mathrm{~b}$ shows the experimental PINEM image of the resulting plasmonic interference pattern (PIP), recorded at a zero relative delay time $\tau$ between the optical pump and electron probe pulse maxima (the chosen arbitrary temporal reference). The spatial fast Fourier transform (FFT) of the entire frame in Fig. $2 \mathrm{~b}$ gives direct access to the spatial frequencies present in the interference pattern ${ }^{8}$, and its radial integral is singularly peaked at the spatial period corresponding to the wavelength of the optically driven SPP wave (Fig. 2c). We can thus directly extract the SPP wavelength $\lambda_{\text {SPP,exp }}$ and wavevector $k_{\mathrm{SPP}}=2 \pi / \lambda_{\mathrm{SPP} \exp }$ as $633 \pm 13 \mathrm{~nm}$ and $9.94 \pm 0.20 \mu \mathrm{m}^{-1}$, respectively, where the errors are due to the uncertainty in the calibration of the PINEM image (Methods). Figure $2 \mathrm{~d}$ depicts the calculated electron energy loss probability for $200 \mathrm{keV}$ electrons transmitted through a model of our layered sample $(30 \mathrm{~nm} \mathrm{Ag}$ on $50 \mathrm{~nm} \mathrm{Si}{ }_{3} \mathrm{~N}_{4}$ ), plotted as a function of energy loss and momentum transfer normal to the beam direction. From the relative EELS probabilities for the different plasmon modes it becomes clear that the electron probe mainly couples to the buried plasmon mode. Given the known energy of the optically driven SPPs in the measurements $\left(E_{\mathrm{SPP}}=E_{\mathrm{photon}}=1.58 \pm 0.015 \mathrm{eV}\right)$, we find that the experimentally obtained SPP characteristics indeed match well with the theoretical dispersion of the plasmonic mode propagating at the buried interface (Supplementary Fig. 4).

Since PINEM is an ultrafast field-of-view technique, it can track the evolution of the photoexcited plasmonic wave in time, given a sufficient combined spatiotemporal resolution. Despite the convolution with finite duration electron bunches, here we can follow its evolution and build-up as it propagates away from the linear NC array on photoexcitation by performing a slice analysis of the PINEM images obtained at different time delays $\tau$. As sketched in Fig. 3a, we divide up each of the different snapshots in a series of horizontal slices centered at increasing distances $y$ from the NC array. By taking the spatial FFT of these slices for each delay, and integrating the Fourier features corresponding to the SPP interference, we can track the propagation of the plasmonic wavefront in time, as is sketched for a number of discrete slices in Fig. 3b. These time traces correspond to the sub-ps cross-correlation of the electron probe 
a

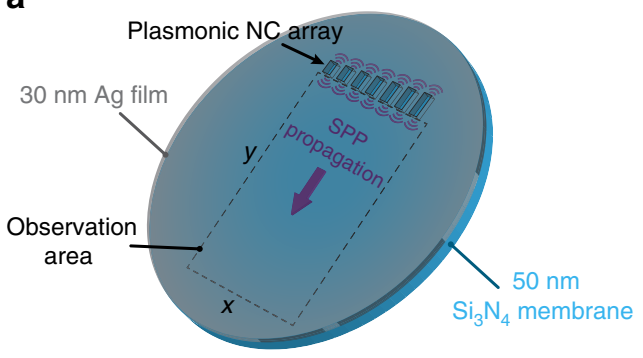

d

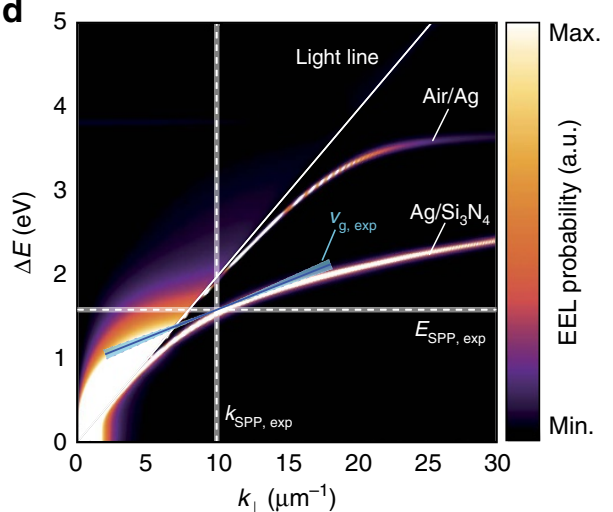

b
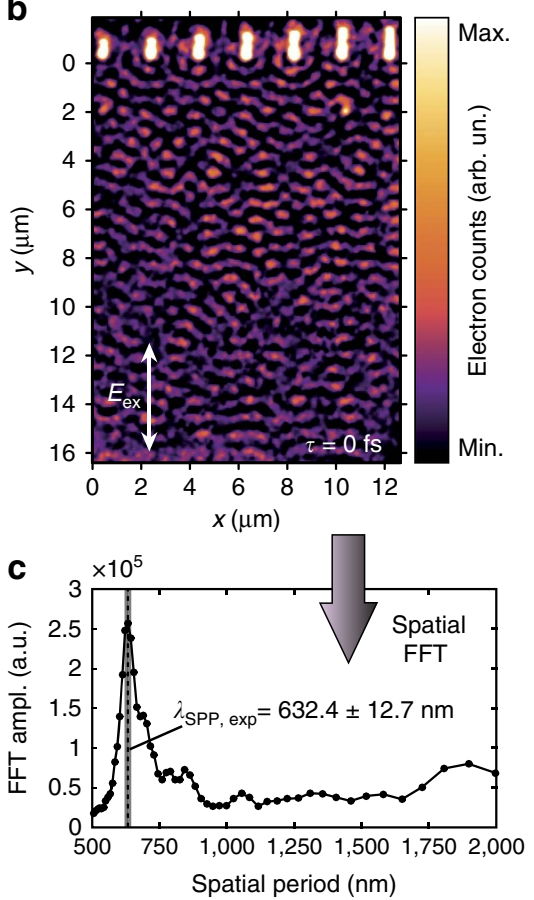

Figure 2 | PINEM imaging of SPP interference at a buried interface. (a) Schematic of the sample and surface plasmon polariton (SPP) propagation experiment. (b) Experimental photon-induced near-field electron microscopy (PINEM) image of the photoinduced plasmonic wave propagating at the buried $\mathrm{Ag} / \mathrm{Si}_{3} \mathrm{~N}_{4}$ interface. The image was recorded at zero delay between electron and laser pulses ( $\tau=0 \mathrm{ps}$ ), using only electrons that have gained energy from the plasmonic near-field. The excitation light was polarized parallel to the long axis of the NCs. As the electron and optical pulse durations satisfy $\Delta_{e}>>\Delta_{p}$, the plasmonic interference pattern (PIP) is observed in the entire window at this delay. The linear false colour scale corresponds to relative electron counts. (c) Radially integrated fast Fourier transform (FFT) amplitude of the spatial frequency components contained in the PIP of $\mathbf{b}$ as a function of the corresponding spatial period (that is, the radial distance in Fourier space). The position of the singular peak, indicated by the dashed line, corresponds to $\lambda_{\text {SPP,exp }}$ and its uncertainty, calculated from the estimated maximum s.d. of the nm-per-pixel calibration value, is indicated by the shaded grey line. (d) Analytically calculated $^{30}$ electron energy loss (EEL) probability of $200 \mathrm{keV}$ electrons traversing a $30 \mathrm{~nm} \mathrm{Ag}$ on $50 \mathrm{~nm} \mathrm{Si}_{3} \mathrm{~N}_{4}$ layer stack (a) at normal incidence, as a function of energy loss $\Delta E$ and transversal momentum transfer $k_{\perp}$ (with respect to the beam direction). Two dispersion branches are observable to the right of the light cone (solid straight line), corresponding to SPP modes propagating along the air/ $\mathrm{Ag}$ and $\mathrm{Ag} / \mathrm{Si}_{3} \mathrm{~N}_{4}$ interfaces, respectively (Supplementary Fig. 4). Experimental magnitudes are indicated by dashed lines (text), with corresponding uncertainties indicated by thick shaded lines. In particular, the solid blue line indicates the measured SPP group velocity $v_{g \text {,exp }}$ (Fig. 3), with the shaded hourglass shape representing the corresponding experimental error.

a

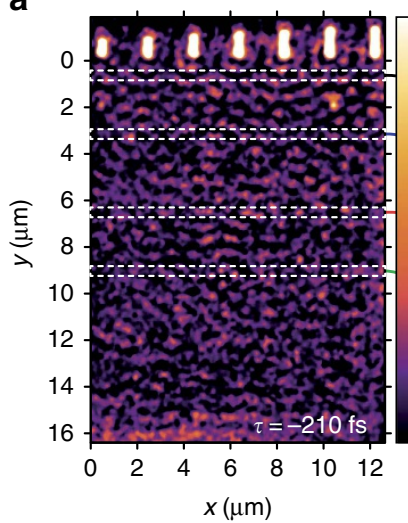

b

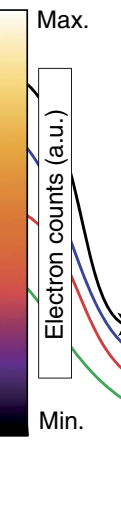

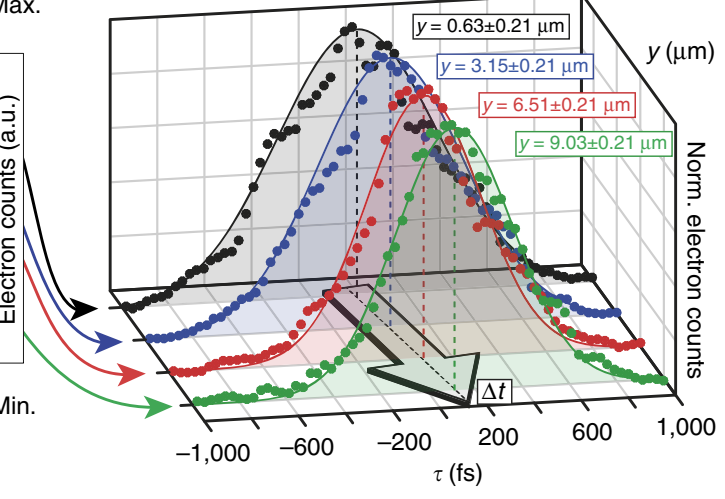

C

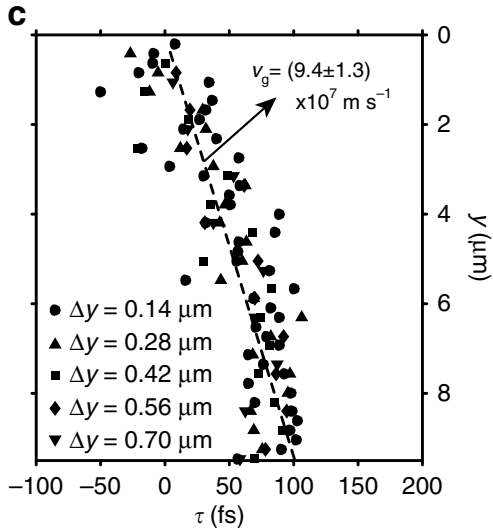

Figure 3 | Direct PINEM measurement of SPP propagation. (a) Experimental photon-induced near-field electron microscopy (PINEM) image of the photoinduced plasmonic wave propagating at the buried $\mathrm{Ag} / \mathrm{Si}_{3} \mathrm{~N}_{4}$ interface. The image was recorded at $\tau=-210 \mathrm{fs}$, using only electrons that have gained energy from the plasmonic near-field. White dashed rectangles exemplify the discrete slicing of the image for spatial Fourier analysis. Electron counts are plotted on a linear false colour scale. (b) Temporal traces of the surface plasmon polariton (SPP) wave intensity in the different discrete slices indicated in a. For each slice at each time delay $\tau$, the SPP wave intensity is calculated as the integrated intensity of the corresponding features in the spatial fast Fourier transform (FFT). The $y$-range of each of the slices corresponds to $\Delta y=0.42 \mu \mathrm{m}$. (c) Temporal centre positions of Gaussian fits to the cross-correlation time traces versus distance travelled by the SPP wave ( $y$ ). Data points corresponding to different slicing schemes (varying $\Delta y$ ) are combined (Methods). The linear slope fitted to the combined data (dashed line) directly yields the group velocity of the SPP wave, $v_{\mathrm{g}, \exp }=(9.4 \pm 1.3) \times 10^{7} \mathrm{~m} \mathrm{~s}-1, \mathrm{corresponding}^{-}$ to an effective SPP refractive index of $c / v_{\text {g,exp }} \simeq 3$. 
and the photoexcited SPP pulse in the different discrete $y$-ranges in the sample, offset by a temporal difference $\Delta t$ due to the SPP wave propagation. Combining the data of all slices yields a quantitative description of the dynamic behaviour, which directly captures the SPP propagation. Figure $3 \mathrm{c}$ depicts the temporal centre of Gaussian fits to the cross-correlation time traces of different slicing schemes as a function of the distance $y$ from the $\mathrm{NC}$ array. The linear slope of this curve is a direct measure of the group velocity of the composite plasmonic wave, corresponding to approximately a third of the free-space speed of light, $v_{\mathrm{g}, \exp }=(9.4 \pm 1.3) \times 10^{7} \mathrm{~m} \mathrm{~s}^{-1}$. This value is consistent with that expected from theory for the buried SPP mode, as indicated in Fig. 2d. The SPP propagation is further visualized in the time-resolved real-space PINEM movie in Supplementary Movie 1 and in Supplementary Fig. 5. SPP group velocities at surfaces and interfaces have been previously extracted from both time domain ${ }^{19,46}$ and frequency domain ${ }^{16,22,47}$ experiments for plasmons whose near-field extends outside of the waveguide material. Nonetheless, a major advantage of the time-resolved PINEM approach is the ability to measure the propagation characteristics even for truly sub-surface plasmons that are entirely buried inside the material, such as for protected graphene waveguides (Supplementary Fig. 6).
Shaping transient plasmonic gratings. The ability of PINEM to directly access PIPs also opens up a multitude of possibilities for creating arbitrarily shaped plasmonic transient gratings, as the obtained interferometric structures can be precisely tailored by combining appropriately arranged nanoscale features with suitable photoexcitation ${ }^{14,44,45,48,49}$. Moreover, experiments can be supported by simulations applying Maxwell's equations to the nanopattern architectures, providing effective feedback and enabling the predictive design of transient PIPs. Figure 4 illustrates this flexibility in designing transient PIPs. Figure $4 \mathrm{a}, \mathrm{b}$ shows the PINEM image of a linear array of long NCs under photoexcitation using different linear polarization orientations, demonstrating that it is indeed possible to obtain transient plasmonic gratings using the interference between counter-propagating plasmons in relatively simple nanostructures. More complex interference patterns can be generated away from such linear NC arrays, as shown for an array formed by short NCs (Fig. 4c). One particularly interesting feature of an SPP wave propagating away from a periodic source array is the plasmon Talbot effect, in which the source configuration is self-imaged into an array of plasmon focal spots at characteristic propagation distances ${ }^{50}$. This is predicted to occur even outside the paraxial regime, and on close inspection
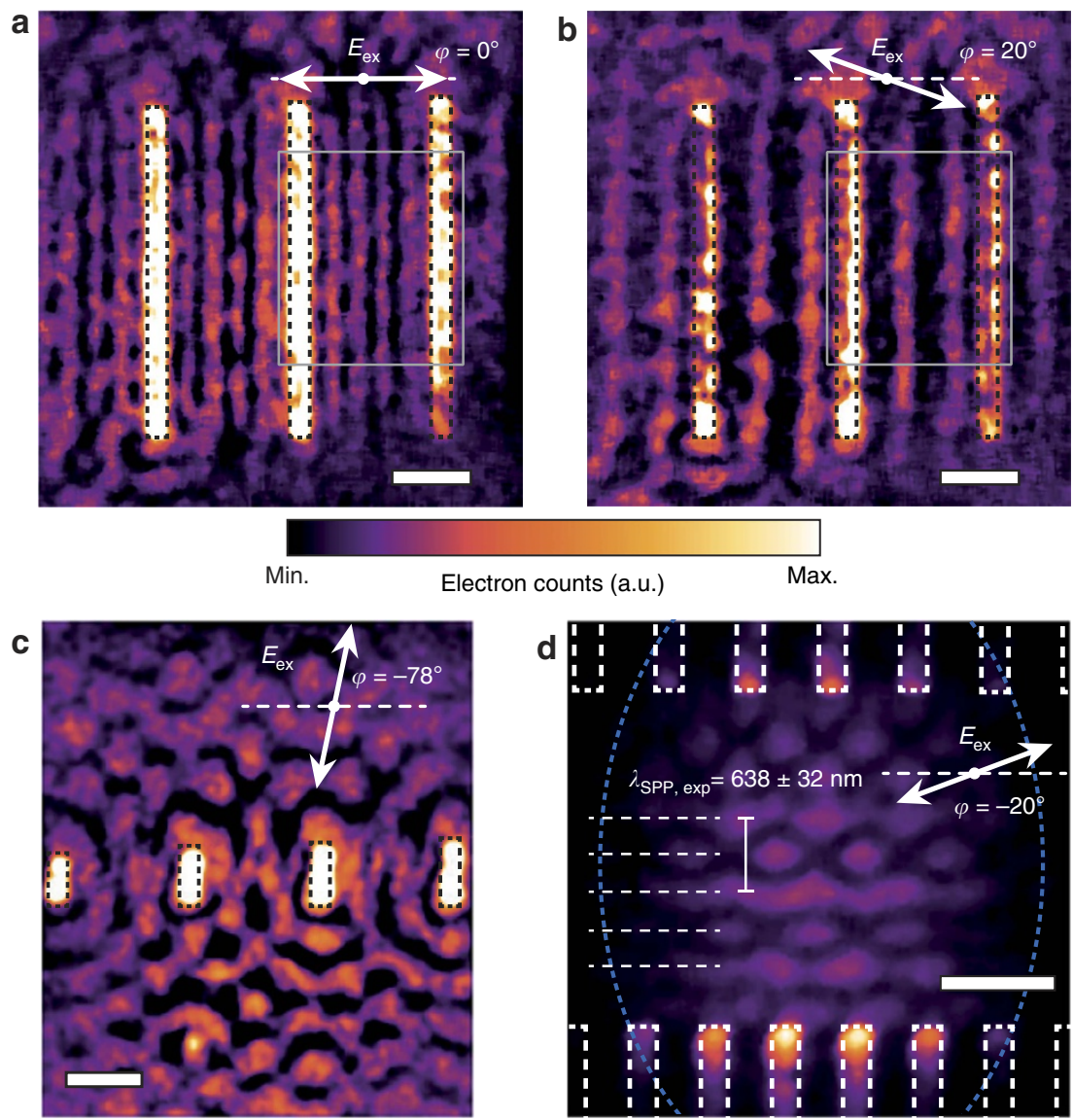

Figure 4 | PINEM imaging of NC-based nanopatterns. Photon-induced near-field electron microscopy (PINEM) images of the transient surface plasmon polariton (SPP) grating generated by a linear array of nanocavities (NCs; measured lengths $\simeq 4.2,4.3$ and $4.4 \mu \mathrm{m}$, respectively, widths $\simeq 260 \mathrm{~nm}$ ), photoexcited by linearly polarized light at $\varphi=0^{\circ}$ and $\varphi=20^{\circ}$, are shown in $\mathbf{a}$ and $\mathbf{b}$, respectively. The area enclosed by the grey rectangle is analysed in detail in Fig. 5. Scale bars in all panels correspond to $1 \mu \mathrm{m}$. (c) PINEM image of the plasmonic interference pattern (PIP) generated by a linear array of short $\mathrm{NCs}$ (measured lengths $\simeq 0.6,0.7,0.8$ and $0.9 \mu \mathrm{m}$, respectively, widths $\simeq 275 \mathrm{~nm}$ ), photoexcited by linearly polarized light at $\varphi=-78^{\circ}$. (d) PINEM image of the PIP generated by two vertically offset linear arrays of NCs (length $\simeq 2.1 \mu \mathrm{m}$, width $\simeq 270 \mathrm{~nm}$ ), photoexcited by linearly polarized light at $\varphi=-20^{\circ}$. The resulting SPP standing wave interference shows a periodicity of $\lambda_{\mathrm{SPP}} / 2$ along the vertical (counter-)propagation direction (dashed horizontal lines), yielding an estimated SPP wavelength of $\lambda_{\text {SPP, exp }}=638 \pm 32 \mathrm{~nm}$. The dashed elliptical shape depicts the approximate outline of the electron beam footprint. All panels are recorded at $\tau=0$ using $\lambda_{0}=786 \mathrm{~nm}$ and share the linear false colour scale depicted in the middle. 
its signature can indeed be seen in Fig. $2 \mathrm{~b}$ at $y \approx 4.2 \mu \mathrm{m}$, corresponding to the half-period shifted revival at half of the socalled Talbot distance. By combining two linear NC arrays with an appropriate relative positioning and photoexcitation, one can exploit this effect even at shorter propagation distances, allowing for the generation of multiple closely spaced arrays of nanoscale focal spots of near-field intensity, and thus charge density, at the buried $\mathrm{Ag} / \mathrm{Si}_{3} \mathrm{~N}_{4}$ interface (Fig. $4 \mathrm{~d}$ ). Here the PIP is dominated by the interference of counter-propagating SPPs generated at the different arrays, such that its $\lambda_{\mathrm{SPP}} / 2$ periodicity along the propagation axis yields $\lambda_{\mathrm{SPP} \text {,exp }}=638 \pm 32 \mathrm{~nm}$, which is consistent with the value extracted in Fig. 2. Given the thin metal layer and relatively narrow NCs, the influence of multiple scattering and reflections of the SPPs on the structure of the resulting standing wave pattern is small, such that a simple analytical model based on a double array of point-dipole SPP sources can reproduce the major features observed (Supplementary Fig. 7). By contrast, this model does not capture the observed complex interference of co-propagating SPPs (as in the propagation experiment). In this case, the time-averaged interference between SPPs from the different point-dipole sources is weaker (similar to that in the plasmon Talbot effect), and additional experimental factors such as non-uniform time-averaging (due to the optical pulse envelope), finite bandwidth excitation and multiple order PINEM scattering are expected to play a dominant role.

As shown in Fig. 4a,b, the shape of the transient SPP grating generated by the linear array of long NCs is strongly dependent on the excitation polarization (Supplementary Movie 2). Figure 5 analyses the indicated region in more detail. In particular, we show in Fig. 5a that on photoexcitation using light polarized perpendicular to the NC long axis, the counter-propagating SPPs between the NCs set up a transient plasmonic grating with a period corresponding to half the SPP wavelength. This yields another independent determination of the SPP carrier wavelength $\left(\lambda_{\mathrm{SPP}, \exp }=639 \pm 6 \mathrm{~nm}\right)$, in good agreement with both previously extracted values and the calculated dispersion for the SPP mode propagating at the $\mathrm{Ag} / \mathrm{Si}_{3} \mathrm{~N}_{4}$ interface. Interestingly, varying the polarization angle of the incident light markedly changes the relative intensities of the near-field grating fringes. At a polarization angle of $\varphi=20^{\circ}$, two of the grating fringes are almost completely suppressed, such that the periodicity of the plasmonic grating has effectively doubled (Supplementary Fig. 8). This is further illustrated in Fig. 5b,c, which depict the full polarization dependence of the transient plasmonic grating. Figure $5 \mathrm{~b}$ plots the fringe contrast $\eta$, calculated as $I_{\text {PINEM,max }} / I_{\text {PINEM }}(\varphi)$, for each of the four fringes indicated in Fig. $5 \mathrm{a}$, revealing a clear intensity dip in the $\varphi$-dependence of peaks 1 and 3 centred around $\varphi=20^{\circ}$. Figure $5 \mathrm{c}$ depicts a Fourier analysis of the SPP grating, with the dominant spatial period of the grating indicated by the red squares (right vertical axis) and the grating amplitude ratio $\rho$ (defined as the ratio of FTT amplitudes $A\left(p_{1}\right) / A\left(p_{2}\right)$ at spatial periods $\left.p_{i}=\lambda_{\mathrm{SPP}} / i\right)$ indicated by the solid black circles (left vertical axis). These results demonstrate that it is possible to reversibly switch the periodicity of the transient plasmonic grating by tuning the light polarization, showing great promise as a potential means for ultrafast switching of localized charge density in quasi-2D materials and heterostructures.

\section{Discussion}

The unique combination of characterization and control capabilities offered by PINEM enables new scenarios for nanoplasmonic circuits. Advanced nanopatterning and multilayered designs could be used to exploit the quantum entanglement of plasmons $^{51,52}$, efficient plasmon sources based on the quantum Cerenkov effect ${ }^{53,54}$ or local nonlinear effects at topological defects $^{55}$, to name a few. In recent years, the emergence of methods to make integrated 2D heterostructures ${ }^{56,57}$ based on graphene $\mathrm{s}^{58}$, hexagonal $\mathrm{BN}^{59}$ and the layered transition metal dichalcogenides ${ }^{60}$, along with the availability of new plasmonic materials $^{61}$, has opened up many possibilities in nanoscale material design. Together, these materials and techniques offer a comprehensive set of tools to develop and advance plasmon-based technology in a wide range of areas, including plasmonic sensing ${ }^{4}$, nonlinear plasmonics ${ }^{62}$ and plasmonic lasers ${ }^{63,64}$. Most, if not all, of these applications are anticipated to make use of protected plasmon modes whose near-fields are confined to remain entirely internal to the material heterostructure, such that the time-resolved PINEM approach discussed here provides the only existing experimental means of spatiotemporal access. Furthermore, the
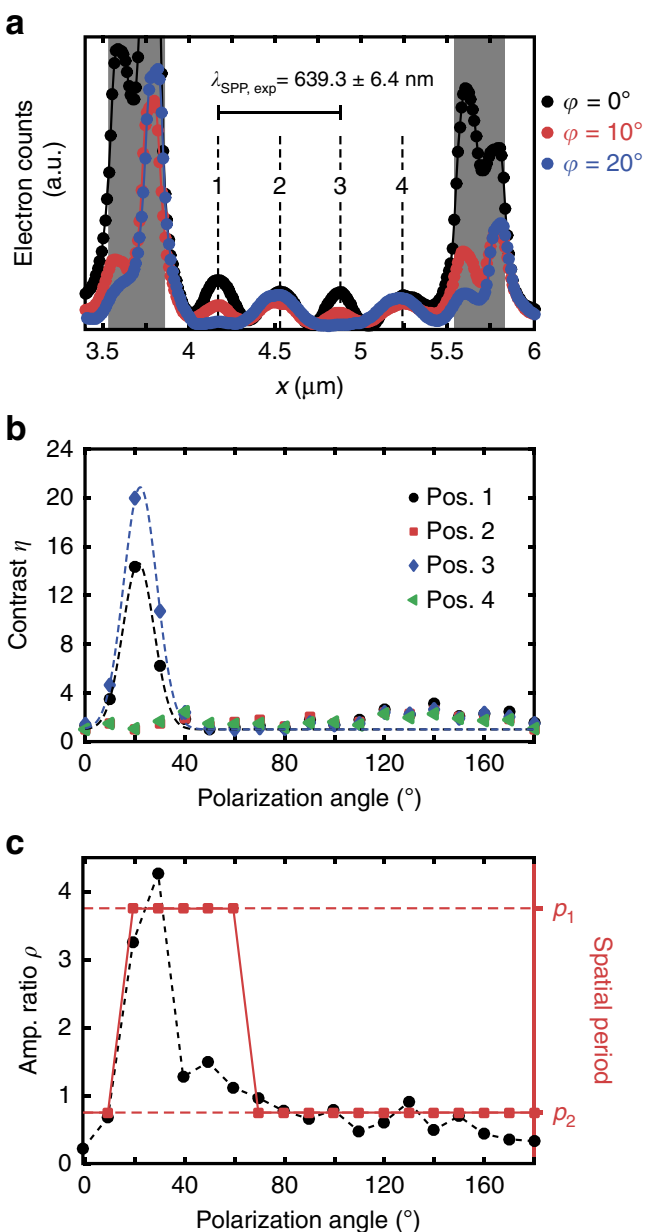

Figure 5 | Polarization control of transient plasmonic gratings. (a) Spatial cross-section of the photon-induced near-field electron microscopy (PINEM) intensity for selected incident polarization angles $\varphi$ (vertically integrated over the area indicated by the rectangle in Fig. 4a,b). The spacing between the numbered fringes corresponds directly to $\lambda_{\mathrm{SPp}} / 2$, yielding an experimental value of $\lambda_{\mathrm{SPP}, \exp }=639 \pm 6.4 \mathrm{~nm}$. (b) Fringe contrast $\eta$, calculated as $I_{\text {PINEM, max }} / I_{\text {PINEM }}(\varphi)$, for each of the four numbered fringes indicated in a. Dashed lines correspond to Gaussian fits. (c) Polarization dependence of the two spatial components in the fast Fourier transform (FFT) spectrum of the surface plasmon polariton (SPP) near-field between the NCs. Black solid circles (left axis) depict the FFT amplitude ratio $\rho$, defined as the ratio of FTT amplitudes $A\left(p_{1}\right) / A\left(p_{2}\right)$ at spatial periods $p_{i}=\lambda_{\mathrm{SPp}} / i$, while the red solid squares indicate the dominant spatial period in the spatial FFT. 
ability to control the spatial shape and temporal evolution of confined plasmonic fields in PINEM enables the implementation of nanoscale experiments to showcase fundamental properties of quantum mechanics ${ }^{39}$ and coherently manipulate electron beams ${ }^{40}$. In analogy to the Kapitza-Dirac effect ${ }^{65,66}$, describing the interaction between photons and electrons in free space, it was recently shown ${ }^{67}$ that electron diffraction from standing plasmon waves can be feasible under experimental conditions similar to those achieved in PINEM, paving the way to the active control of electron diffraction for the generation of tailored beams (for example, vortex beams) and other exotic superpositions of electron wave functions.

Summarizing, in this work we demonstrate the in situ visualization of photoinduced plasmonic interference patterns confined to otherwise inaccessible buried interfaces, enabling a critical tool for the investigation and development of complex plasmonic heterostructures and advanced multilayer devices. Our experiments demonstrate the feasibility of ultrafast imaging of plasmon dynamics using PINEM, allowing us to experimentally measure the carrier wavelength and propagation speed of SPPs travelling along buried interfaces directly in the time domain. Furthermore, we show that transient plasmonic interference patterns can be shaped, manipulated and controlled through both the polarization of the excitation light and the nanopatterning architecture, thus facilitating a widely tunable range of nanoscale near-field structures. Finally, the results presented here represent a considerable advance towards the realization of the recently proposed methodology involving inelastic electron diffraction from transient plasmonic gratings.

\section{Methods \\ Materials. A silver thin film was sputtered onto an in-house fabricated} $\mathrm{Si}_{3} \mathrm{~N}_{4}$-on-Si support using an EMITECH K575x sputter coater equipped with an $\mathrm{Ag}$ target $\left(100 \mathrm{~mA}, 60 \mathrm{~s}\right.$ exposure, $3 \times 10^{-4} \mathrm{mbar}$ base pressure). The nominal thickness of the $\mathrm{Si}_{3} \mathrm{~N}_{4}$ membrane was $50 \mathrm{~nm}$, with potential thickness variation between 35 and $50 \mathrm{~nm}$. Nanopatterns were written in the Ag layer using a raster-scanned focused-ion beam (FEI Nova Nanolab 200 focused ion beam/scanning electron microscope) with typical beam currents of $9-10 \mathrm{pA}$ at a $30 \mathrm{kV}$ voltage. Supplementary Fig. 3b,c shows an SEM characterization of the sample, which was kept in low-oxygen conditions and measured in the ultrafast transmission electron microscope (UTEM), at $295 \mathrm{~K}$ and $\sim 10^{-5} \mathrm{~Pa}$ ) within a few days of patterning in the FIB. For the propagation experiment, nominal NC widths were $250 \mathrm{~nm}$ (experimentally measured (SEM) widths $\simeq 274 \mathrm{~nm}$ ) with nominal NC lengths incrementing from 700 to $1,300 \mathrm{~nm}$ in $100 \mathrm{~nm}$ steps (from left to right, NCs in field of view in Fig. 3a, experimentally measured (SEM) lengths ( $\pm 0.3 \%)$ : 698, $809,919,982,1,113,1,175$ and $1,273 \mathrm{~nm})$.

Experimental. A $300 \mathrm{kHz}$ train of linearly polarized light pulses was split to yield two beams, one of which was frequency-tripled to deliver few-nJ ultraviolet pulses $(262 \mathrm{~nm})$ to a custom truncated-cone $\mathrm{LaB}_{6}$ photo-cathode $(15 \mu \mathrm{m}$ diameter flat tip, produced by AP-Tech) in a custom-modified UTEM ${ }^{37}$. The ultraviolet pulse train was attenuated to range average power to ensure photoemission of ultrafast $200 \mathrm{keV}$ single-electron bunches, minimizing electron-electron interactions in the probe and yielding an optimized beam coherence at an electron bunch duration of $\sim 650$ fs FWHM (bunch envelope proportional to $e^{-\left(\frac{t+\tau}{\Delta_{e}}\right)^{2}}$, where $\tau$ is the relative time delay of the electron bunch with respect to the optical pump pulse, Fig. 1). The second optical beam (FWHM duration $\sim 105 \mathrm{fs}$, central wavelength $\lambda_{0}=786 \pm 15 \mathrm{~nm}$ ), after undergoing a variable delay, was weakly focused on the sample in the UTEM at near-normal incidence using an achromatic doublet $(f=250 \mathrm{~mm})$ to a spot size of $\simeq 100 \mu \mathrm{m}$ diameter at the sample plane, such that the electric field of the pump beam was uniform across the field of view of the electron beam. The time delay $\tau$ is defined as the arrival time of the midway point of the Gaussian electron probe pulse at the sample $z$-position with respect to that of the Gaussian optical pump pulse. Optical pump fluences employed were of the order of $1-4 \mathrm{~mJ} \mathrm{~cm}^{-2}$, corresponding to a peak excitation energy density of $\sim 50 \mathrm{GW} \mathrm{cm}{ }^{-2}$. Under these experimental conditions, the generation of SPPs by the transient electric field associated with the electron probe beam is a much weaker effect, which can be considered negligible ${ }^{34}$. A detailed description and characterization of the modified JEOL JEM 2100 microscope can be found elsewhere ${ }^{37}$. For the PINEM experiments described in this work, the UTEM was operated at $200 \mathrm{keV}$ in photoelectron mode. The Gatan imaging filter (GIF) camera was operated with a $0.05 \mathrm{eV}$-per-channel dispersion setting, while typical exposure times of the $2,048 \times 2,048$ pixel CCD sensor were $60 \mathrm{~s}$ for images and $10 \mathrm{~s}$ for spectra. Electron energy loss spectra were aligned based on their zero loss peak positions using a differential-based maximum intensity alignment algorithm.

Image processing. Variable delay PINEM frames were spatially aligned using a custom video stabilization algorithm. All obtained PINEM images were median-filtered for single pixel noise and spike removal, and background subtracted using a high-pass spatial-frequency Fourier filter set to remove contributions corresponding to spatial periods larger than $\simeq 5 \mu \mathrm{m}$. PINEM image calibration was done using the SEM-measured NC lengths in the field of view as independent references, with the final nm-per-pixel parameter taken as the mean of the calibration factors independently determined for the different NCs. The resulting uncertainty in the image scale is the principal contribution to the error bars on the extracted $\lambda_{\mathrm{SPP}, \exp }$ and $k_{\mathrm{SPP} \text {,exp }}$ values. The fringe positions in Figs $4 \mathrm{~d}$ and $5 \mathrm{a}$ were determined by fitting Gaussian lineshapes to the corresponding integrated spatial traces (the image in Fig. $4 \mathrm{~d}$ was horizontally integrated), and the resulting $\lambda_{\mathrm{SPP} \text {,exp }}$ values were calculated from the average fringe spacing. Light polarization angles $\varphi$ at the sample position inside the UTEM were determined to be within a systematic error of $\pm 5^{\circ}$ and verified by comparison with simulations of the plasmonic field.

For a robust determination of the SPP group velocity, the positive $y$-range of PINEM images recorded at different time delays $\tau$ were divided up into a series of discrete horizontal slices, according to various slicing schemes. The data in Fig. 3b correspond to the spatial FFT analysis of a series of slices with a $y$-range of $\Delta y=0.42 \mu \mathrm{m}$. For each slice, a Gaussian temporal profile was fitted to the $\tau$-dependence of the integrated intensity of the spatial FFT features characteristic of the SPP interference. Full slicing analyses were carried out for slicing schemes having a $\Delta y=0.14,0.28,0.42,0.56$ and $0.70 \mu \mathrm{m}$, in each case with a $y$-centre offset equal to $0.5 \Delta y$ ( $50 \%$ slice overlap). The resulting data points (fitted temporal Gaussian centre versus $y$-range centre) for each of the slices in the different slicing schemes were combined and fitted with a single straight line in the initial SPP propagation range $(y=0.1-9.7 \mu \mathrm{m})$, where the slope represents the group velocity of the propagating plasmonic wave (Fig. 3c).

Analytical calculations. The dispersion diagrams in Fig. $2 \mathrm{~d}$ and Supplementary Fig. 6a represent the energy-loss- and in-plane momentum-transfer-resolved loss probability experienced by an electron crossing the $\mathrm{Ag} / \mathrm{Si}_{3} \mathrm{~N}_{4}$ and $\mathrm{Si} /$ graphene/Si structures of the samples under normal incidence. The probability is calculated analytically using the following methods ${ }^{30}$. In brief, the total energy loss is first obtained by integrating along the trajectory of the retarding force produced by the induced electronic field that is created by the electron and acting back on it. Then the result is expressed as a double integral over energy- $(\Delta E)$ and momentumtransfer $\left(k_{\perp}\right)$ components, which are interpreted as $\Delta E$ times the energy- andmomentum-resolved distribution of the loss probability (that is, the EEL probability). The induced field is obtained by considering a source field produced by the electron in each of the media as though it was moving in an extended homogeneous material. This source field is then scattered at each interface, producing transverse magnetic reflected waves. Note that the source field does not couple to transverse electric waves due to the $m=0$ axial symmetry of the problem for normal electron incidence. The amplitudes of the reflected waves are subsequently determined by the continuity of the electric field and the normal displacement, and finally the integral of the retarding force along the trajectory is carried out analytically within each material for each combination of $\Delta E$ and $k_{\perp}$. The materials $\left(\mathrm{Ag}, \mathrm{Si}_{3} \mathrm{~N}_{4}\right.$ and $\left.\mathrm{Si}\right)$ are represented through their dielectric functions, taken from tabulated measured data ${ }^{68,69}$. The dielectric response of graphene is described using a Drude model ${ }^{70}$ with realistic parameters for the Fermi energy $(0.5 \mathrm{eV})$, layer thickness $(0.3 \mathrm{~nm})$ and damping $(1.0 \mathrm{meV})$.

An approximate plasmonic interference pattern for the nanostructure imaged in Fig. 4d was analytically calculated based on arrays of simple point-dipole SPP sources (Supplementary Fig. 7). The model takes into account the finite propagation distance $\gamma \approx 64 \mu \mathrm{m}$, defined as the $1 / e$ decay distance of the plasmon electric field, calculated from the tabulated permittivities for SPPs at a $\mathrm{Ag} / \mathrm{Si}_{3} \mathrm{~N}_{4}$ interface (semi-infinite slabs) at the photon energy under consideration. This value is consistent with the experimental observations in this work, which show little spatial plasmonic field decay within the field of view. All sources were assumed to be monochromatic $\left(\lambda_{\mathrm{SPP}}=638 \mathrm{~nm}\right)$, radiating SPP waves travelling radially outward in a dipolar pattern. All dipoles were oriented along the same direction (maximum radiated field along $\varphi=-20^{\circ}$, with the point dipole oscillating along the perpendicular direction, that is, $\varphi=70^{\circ}$ ). The resulting complex $E_{z}$-field components originating from the different sources were coherently summed, after which uniform time-averaging was incorporated by taking the square modulus of the sum.

Data availability. The data that support the findings of this study are available on request from the corresponding authors. 


\section{References}

1. Maier, S. A. in Plasmonics: Fundamentals and Applications 1st edn (Springer, 2007).

2. Barnes, W. L., Dereux, A. \& Ebbesen, T. W. Surface plasmon subwavelength optics. Nature 424, 824-830 (2003).

3. Ozbay, E. Plasmonics: merging photonics and electronics at nanoscale dimensions. Science 311, 189-193 (2006).

4. Stewart, M. E. et al. Nanostructured plasmonic sensors. Chem. Rev. 108, 494-521 (2008).

5. Liu, X., Wang, Y. \& Potma, E. O. A dual-color plasmonic focus for surface-selective four-wave mixing. Appl. Phys. Lett. 101, 081116 (2012).

6. Bohm, C., Bangert, J., Mertin, W. \& Kubalek, E. Time resolved near-field scanning optical microscopy. J. Phys. D Appl. Phys. 27, 2237-2240 (1994).

7. Zayats, A. V., Smolyaninov, I. I. \& Maradudin, A. A. Nano-optics of surface plasmon polaritons. Phys. Rep. 408, 131-314 (2005).

8. Gersen, H. et al. Real-space observation of ultraslow light in photonic crystal waveguides. Phys. Rev. Lett. 94, 073903 (2005)

9. Wagner, M. et al. Ultrafast dynamics of surface plasmons in InAs by time-resolved infrared nanospectroscopy. Nano Lett. 14, 4529-4534 (2014).

10. Nishiyama, Y., Imura, K. \& Okamoto, H. Observation of plasmon wave packet motions via femtosecond time-resolved near-field imaging techniques. Nano Lett. 15, 7657-7665 (2015).

11. Petek, H. \& Ogawa, S. Femtosecond time-resolved two-photon photoemission studies of electron dynamics in metals. Prog. Surf. Sci. 56, 239-310 (1997)

12. Kubo, A. et al. Femtosecond imaging of surface plasmon dynamics in a nanostructured silver film. Nano Lett. 5, 1123-1127 (2005).

13. Kubo, A., Pontius, N. \& Petek, H. Femtosecond microscopy of surface plasmon polariton wave packet evolution at the silver/vacuum interface. Nano Lett. 7, 470-475 (2007).

14. Aeschlimann, M. et al. Spatiotemporal control of nanooptical excitations. Proc. Natl Acad. Sci. USA 107, 5329-5333 (2010).

15. Lemke, C. et al. Spatiotemporal characterization of SPP pulse propagation in two-dimensional plasmonic focusing devices. Nano Lett. 13, 1053-1058 (2013).

16. Lemke, C. et al. The interplay between localized and propagating plasmonic excitations tracked in space and time. Nano Lett. 14, 2431-2435 (2014).

17. Gong, Y., Joly, A. G., Hu, D., El-Khoury, P. Z. \& Hess, W. P. Ultrafast imaging of surface plasmons propagating on a gold surface. Nano Lett. 15, 3472-3478 (2015).

18. Mårsell, E. et al. Nanoscale imaging of local few-femtosecond near-field dynamics within a single plasmonic nanoantenna. Nano Lett. 15, 6601-6608 (2015).

19. Lemke, C. et al. Mapping surface plasmon polariton propagation via counter-propagating light pulses. Opt. Express 20, 12877-12884 (2012)

20. Lemke, C. et al. Measurement of surface plasmon autocorrelation functions. Opt. Express 21, 27392-27401 (2013).

21. Bozhevolnyi, S. I., Smolyaninov, I. I. \& Zayats, A. V. Near-field microscopy of surface-plasmon polaritons: localization and internal interface imaging. Phys. Rev. B 51, 17916-17924 (1995).

22. Leißner, T. et al. Morphological tuning of the plasmon dispersion relation in dielectric-loaded nanofiber waveguides. Phys. Rev. Lett. 111, 046802 (2013).

23. Lemke, C. et al. The complex dispersion relation of surface plasmon polaritons at gold/para-hexaphenylene interfaces. Appl. Phys. B 116, 585-591 (2014).

24. Bauer, M. et al. Phase propagation of localized surface plasmons probed by time-resolved photoemission electron microscopy. Appl. Phys. A 88, 473-480 (2007).

25. Kahl, P. et al. Normal-incidence photoemission electron microscopy (ni-peem) for imaging surface plasmon polaritons. Plasmonics 9, 1401-1407 (2014).

26. Kociak, M., Stéphan, O., Walls, M. G., Tencé, M. \& Colliex, C. Scanning Transmission Electron Microscopy: Imaging and Analysis, chap. Spatially Resolved EELS: The Spectrum-Imaging Technique and Its Applications 163-205 (Springer, 2011)

27. Bosman, M., Keast, V. J., Watanabe, M., Maaroof, A. I. \& Cortie, M. B. Mapping surface plasmons at the nanometre scale with an electron beam. Nanotechnology 18, 165505 (2007)

28. Nelayah, J. et al. Mapping surface plasmons on a single metallic nanoparticle. Nat. Phys. 3, 348-353 (2007).

29. Schaffer, B., Hohenester, U., Trügler, A. \& Hofer, F. High-resolution surface plasmon imaging of gold nanoparticles by energy-filtered transmission electron microscopy. Phys. Rev. B 79, 041401 (2009).

30. García de Abajo, F. J., Rivacoba, A., Zabala, N. \& Yamamoto, N. Boundary effects in Cherenkov radiation. Phys. Rev. B 69, 155420 (2004)

31. Rossouw, D. \& Botton, G. A. Plasmonic response of bent silver nanowires for nanophotonic subwavelength waveguiding. Phys. Rev. Lett. 110, 066801 (2013).

32. Barwick, B., Flannigan, D. J. \& Zewail, A. H. Photon-induced near-field electron microscopy. Nature 462, 902-906 (2009).

33. García de Abajo, F. J., Asenjo-Garcia, A. \& Kociak, M. Multiphoton absorption and emission by interaction of swift electrons with evanescent light fields. Nano Lett. 10, 1859-1863 (2010).
34. Park, S. T., Lin, M. \& Zewail, A. H. Photon-induced near-field electron microscopy (PINEM): theoretical and experimental. New J. Phys. 12, 123028 (2010).

35. Park, S. T. \& Zewail, A. H. Photon-induced near-field electron microscopy: mathematical formulation of the relation between the experimental observables and the optically driven charge density of nanoparticles. Phys. Rev. A 89, 013851 (2014).

36. Asenjo-Garcia, A. \& García de Abajo, F. J. Plasmon electron energy-gain spectroscopy. New J. Phys. 15, 103021 (2013).

37. Piazza, L. et al. Design and implementation of a fs-resolved transmission electron microscope based on thermionic gun technology. Chem. Phys. 423 79-84 (2013)

38. Barwick, B. \& Zewail, A. H. Photonics and plasmonics in 4D ultrafast electron microscopy. ACS Photon. 2, 1391-1402 (2015)

39. Piazza, L. et al. Simultaneous observation of the quantization and the interference pattern of a plasmonic near-field. Nat. Commun. 6, 6407 (2015).

40. Feist, A. et al. Quantum coherent optical phase modulation in an ultrafast transmission electron microscope. Nature 521, 200-203 (2015).

41. Liu, H., Baskin, J. S. \& Zewail, A. H. Infrared PINEM developed by diffraction in 4D UEM. Proc. Natl Acad. Sci. USA 113, 2041-2046 (2016).

42. Hassan, M. T., Liu, H., Baskin, J. S. \& Zewail, A. H. Photon gating in four dimensional ultrafast electron microscopy. Proc. Natl Acad. Sci. USA 112, 12944-12949 (2015).

43. Lalanne, P., Hugonin, J. P. \& Rodier, J. C. Approximate model for surface-plasmon generation at slit apertures. J. Opt. Soc. Am. A 23, 1608-1615 (2006).

44. Lin, J. et al. Polarization-controlled tunable directional coupling of surface plasmon polaritons. Science 340, 331-334 (2013).

45. Tanemura, T. et al. Multiple-wavelength focusing of surface plasmons with a nonperiodic nanoslit coupler. Nano Lett. 11, 2693-2698 (2011)

46. Bai, M. et al. Measuring the speed of a surface plasmon. Phys. Rev. B 69, 115416 (2004).

47. Temnov, V. V., Woggon, U., Dintinger, J., Devaux, E. \& Ebbesen, T. W. Surface plasmon interferometry: measuring group velocity of surface plasmons Opt. Lett. 32, 1235-1237 (2007).

48. Aeschlimann, M. et al. Adaptive subwavelength control of nano-optical fields. Nature 446, 301-304 (2007).

49. Aeschlimann, M. et al. Optimal open-loop near-field control of plasmonic nanostructures. New J. Phys. 14, 033030 (2012).

50. Dennis, M. R., Zheludev, N. I. \& García de Abajo, F. J. The plasmon Talbot effect. Opt. Express 15, 9692-9700 (2007).

51. Altewischer, E., van Exter, M. P. \& Woerdman, J. P. Plasmon-assisted transmission of entangled photons. Nature 418, 304-306 (2002).

52. Fakonas, J. S., Lee, H., Kelaita, Y. A. \& Atwater, H. A. Two-plasmon quantum interference. Nat. Photon. 8, 317-320 (2014).

53. Kaminer, I. et al. Efficient plasmonic emission by the quantum Čerenkov effect from hot carriers in graphene. Nat. Commun. 7, 11880 (2016).

54. Genevet, P. et al. Controlled steering of Cherenkov surface plasmon wakes with a one-dimensional metamaterial. Nat. Nanotechnol. 10, 804-809 (2015).

55. Yin, X. et al. Edge nonlinear optics on a $\mathrm{MoS}_{2}$ atomic monolayer. Science 344, 488-490 (2014).

56. Britnell, L. et al. Strong light-matter interactions in heterostructures of atomically thin films. Science 340, 1311-1314 (2013).

57. Gong, Y. et al. Vertical and in-plane heterostructures from $\mathrm{ws}_{2} / \mathrm{mos}_{2}$ monolayers. Nat. Mater. 13, 1135-1142 (2014).

58. Novoselov, K. S. et al. A roadmap for graphene. Nature 490, 192-200 (2012)

59. Pakdel, A., Bando, Y. \& Golberg, D. Nano boron nitride flatland. Chem. Soc. Rev. 43, 934-959 (2014).

60. Wang, Q. H., Kalantar-Zadeh, K., Kis, A., Coleman, J. N. \& Strano, M. S. Electronics and optoelectronics of two-dimensional transition metal dichalcogenides. Nat. Nanotechnol. 7, 699-712 (2012).

61. Wang, Y., Capretti, A. \& Dal Negro, L. Wide tuning of the optical and structural properties of alternative plasmonic materials. Opt. Mater. Express 5, 2415-2430 (2015).

62. Kauranen, M. \& Zayats, A. V. Nonlinear plasmonics. Nat. Photon. 6, 737-748 (2012).

63. Oulton, R. F. et al. Plasmon lasers at deep subwavelength scale. Nature 461, 629-632 (2009).

64. Zhou, W. et al. Lasing action in strongly coupled plasmonic nanocavity arrays Nat. Nanotechnol. 8, 506-511 (2013).

65. Kapitza, P. L. \& Dirac, P. A. M. The reflection of electrons from standing light waves. Proc. Camb. Phil. Soc. 29, 297-300 (1933).

66. Freimund, D. L., Aflatooni, K. \& Batelaan, H. Observation of the Kapitza-Dirac effect. Nature 413, 142-143 (2001).

67. García de Abajo, F. J., Barwick, B. \& Carbone, F. Electron diffraction by plasmon waves. Phys. Rev. B 94, 041404 (2016). 
68. Johnson, P. B. \& Christy, R. W. Optical constants of the noble metals. Phys. Rev. B 6, 4370-4379 (1972).

69. Palik, E. D. (ed.) in Handbook of Optical Constants of Solids (Academic Press Limited, 1998).

70. García de Abajo, F. J. \& Manjavacas, A. Plasmonics in atomically thin materials. Faraday Discuss. 178, 87-107 (2015).

\section{Acknowledgements}

Work at LUMES was supported by ERC starting grant USED258697 (F.C.), Marie Curie IEF grant 623982 (T.T.A.L.) and the NCCR MUST, a research instrument of the Swiss National Science Foundation (SNSF). B.B. was supported by a Trinity College FRC grant and by a CT Space grant. F.J.G.A. was supported by the Spanish MINECO (MAT2014-59096-P and SEV-2015-0522).

\section{Author contributions}

T.T.A.L., G.B. and B.B. conducted experiments, R.J.L. and D.M. fabricated samples, T.L. contributed to apparatus design and optimization, T.T.A.L. and F.J.G.A. performed calculations, T.T.A.L., L.D.N., F.J.G.d.A. and F.C. interpreted results, F.C. conceived and designed the research, T.T.A.L., B.B. and F.C. wrote the article. All authors have read and approved the final manuscript.

\section{Additional information}

Supplementary Information accompanies this paper at http://www.nature.com/ naturecommunications

Competing financial interests: The authors declare no competing financial interests.

Reprints and permission information is available online at http://npg.nature.com/ reprintsandpermissions/

How to cite this article: Lummen, T. T. A. et al. Imaging and controlling plasmonic interference fields at buried interfaces. Nat. Commun. 7, 13156 doi: $10.1038 /$ ncomms13156 (2016).

\section{(c) (i)}

This work is licensed under a Creative Commons Attribution 4.0 International License. The images or other third party material in this article are included in the article's Creative Commons license, unless indicated otherwise in the credit line; if the material is not included under the Creative Commons license, users will need to obtain permission from the license holder to reproduce the material. To view a copy of this license, visit http://creativecommons.org/licenses/by/4.0/

(C) The Author(s) 2016 